Irish Math. Soc. Bulletin

Number 68, Winter 2011, 55-63

ISSN 0791-5578

\title{
A SOLUTION OF $\Delta u+f(u)=0$ ON A TRIANGLE
}

\author{
JOSEPH A. CIMA AND WILLIAM DERRICK
}

\begin{abstract}
We use moving planes and thin domain maximum principles to prove the maximum value of a positive solution to the equation $\Delta u+f(u)=0$ on a symmetric-convex domain $\Omega$, with $u=0$ on the boundary of $\Omega$, lies on the line of symmetry of the domain. If the domain has two or more lines of symmetry the maximum is at their intersection.
\end{abstract}

\section{INTRODUCTION}

In the last several years two novel tools used in the theory of Partial Differential Equations (PDE) have yielded interesting information: the use of "moving planes" and the concept of "maximal principles for thin domains". These ideas have been incorporated in the works of the authors Berestycki, Nirenberg, Varadhan, Ni, Gidas, Fraenkel and Du (see the papers [1]-[3] and [5]). We refer the reader to those papers for more detail on what we will address in this paper, including a very detailed account of some of their work in the book of Fraenkel [4]. Many of these results are based on solutions of an elliptic PDE on a certain special class of bounded domains in $\mathbb{R}^{n}$. We will work only in $\mathbb{R}^{2}$ and we will define the special properties of these domains in the following section. We will call these domains "symmetric-convex" and use the notation S-C for any domain that satisfies the appropriate conditions.

One of the key results of the above papers and books is to prove that the directional derivatives of the solutions in the convex directions are negative when one leaves the line of symmetry and approaches the boundary in the convex direction. The purpose of this paper is to study the location of the critical points where the solution takes its maximum. In general, if the domain in question has

2010 Mathematics Subject Classification. 35?02, 35B50.

Key words and phrases. Maximum Principle, Moving Planes, SymmetricConvex Domains.

Received on 18-2-2011; revised 9-12-2011. 
two possible directions of symmetry then there is a definitive answer to the question. If not, then one knows only that the maximum is taken on the intersection of the line (plane) of symmetry interior to the domain. We will show in a basic case (an isosceles triangle) that the maximum must be achieved on a fixed subset of this line of symmetry. Further, we will include computational work that indicates the solution must be unique and in a significant way is independent of the given function $f(u)$ for the problem.

\section{Definitions And the Methods}

First we define a symmetric-convex (S-C) domain:

Definition 1.1. Let $\Omega$ be a bounded, simply-connected domain in the plane, and assume $m$ and $k$ are unit vectors in the plane which are orthogonal. For each point $P$ on the boundary of $\Omega$ let $L_{P}$ be the subset of $\Omega$ consisting of the points that lie on the line $\{P+r m$ : $r \in \mathbb{R}\}$, i.e.

$$
L_{P}=\Omega \cap(P+\mathbb{R} m) .
$$

Similarly, for $Q$ on $\partial \Omega$, let

$$
L_{Q}^{\prime}=\Omega \cap(Q+\mathbb{R} k) .
$$

The domain $\Omega$ is said to be $\mathrm{S}-\mathrm{C}$ (with respect to $(m, k)$ ) if each nonempty $L_{Q}^{\prime}$ (for $Q \in \partial \Omega$ ) is a segment and there is a point $P_{0} \in \partial \Omega$ for which $L_{P_{0}}$ is a segment and $\Omega$ is symmetric under orthogonal reflection in this segment, i.e. the segment $L_{P_{0}}$ bisects every nonempty $L_{Q}^{\prime}$

For example, a disc is $\mathrm{S}-\mathrm{C}$ for any two orthogonal directions while a Star of David with the standard orientation (i.e., with center of the star at the origin and the $y$ axis joining two opposite points of the star) has three $\mathrm{S}-\mathrm{C}$ orientations. One is the $m$ vector with $\theta=90^{\circ}$ and the other two are for the angles $\theta= \pm 30^{\circ}$.

Let $\Omega$ be a connected bounded S-C domain where, for simplicity, we will assume $U$ is the vector $(1,0)$ and $V$ is $(0,1)$.

We sketch the way the "moving planes" and "thin domain" tools are used in the proofs of the above mentioned authors. For simplicity, consider the strictly elliptic, second order operator on the domain $\Omega$ :

$$
L=\Delta+c(x, y) .
$$

The term $c(\cdot)$ is assumed uniformly bounded by a number, say $c_{0}$. 
Definition 1.2. A maximum principle holds for $L$ in $\Omega$ if

$$
L w \geq 0
$$

in $\Omega$, with $w \in C^{2}(\Omega) \cap C(\bar{\Omega})$, and

$$
w(x, y) \leq 0
$$

on $\partial \Omega$, implies that $w(x, y) \leq 0$, for all $(x, y) \in \Omega$.

Varadhan observes the following:

Proposition 1.3. (Thin Domain Principle.) Assume diam $(\Omega) \leq$ $d$. There exists $\delta>0$ depending only on $d, c_{0}$, such that the maximum principle holds for $L$ in $\Omega$ provided meas $(\Omega)=|\Omega|<\delta$.

Let $u: \bar{\Omega} \rightarrow \mathbb{R}$ be a positive solution of

$$
\Delta u+f(u)=0, \quad u=0 \text { on } \partial \Omega,
$$

with $u \in C(\bar{\Omega}) \cap C^{2}(\Omega)$, where $f:[0, \infty] \rightarrow R$ is $C^{1}$ (thus also Lipschitz) and monotone increasing. We will give a brief overview of how the maximum principles above and the moving plane methods fit together. Assume $0 \in \Omega$ without loss of generality. Then a line $L_{a}=[(x, y): x=a]$ meets the domain $\Omega$ if $a$ is small in modulus. In particular, $L_{0}$ is the $y$ axis. Since the domain $\Omega$ is assumed to be bounded there is a number $a$ such that the line $L_{a}$ meets $\Omega$ and cuts a small "cap" (an open subset of $\Omega$ ), say $\Sigma(a)=\{(x, y) \in \Omega \mid x<a\}$ from $\Omega$.

We examine only one component of this open subset of $\Omega$ and assume $a<0$. Reflecting the domain $\Sigma(a)$ about $L_{a}$, we see that the reflected domain $\Sigma(a)^{\perp} \subset \Omega$, each point $P \in \Sigma(a)$ having a reflected point $P^{\perp} \in \Sigma(a)^{\perp}$. Define

$$
w(P ; a) \equiv u(P)-u\left(P^{\perp}\right)
$$

for $P \in \overline{\Sigma(a)}$. Since $f$ is Lipschitz $w$ satisfies $\Delta w+\gamma(P ; a) w=0$, on $\Sigma(a)$, with

$$
\gamma(P, a)= \begin{cases}\frac{f(u(P))-f\left(u\left(P^{\perp}\right)\right)}{u(P)-u\left(P^{\perp}\right)}, & P \in \Sigma(a), \\ 0, & P \in L_{a} .\end{cases}
$$

By the Maximum principle (or thin domain principle), it follows that $w \leq 0$ in $\overline{\Sigma(a)}$. Next, using a strong maximum principle (see [4, Theorem 2.13]), we extend this to $w<0$ on $\Sigma(a)$.

This leads to the key inequality $u(P)<u\left(P^{\perp}\right)$ for all $P$ in $\Sigma(a)$. Finally, this shows that $u_{x}(P)>0$ in $\Sigma(a)$. Consider the largest 
(non-positive) value of this parameter $a$ for which this is true. If this value is 0 , then one can use symmetry to show, by replacing the argument in the "opposite" direction, that in fact the solution $u(p)$ is strictly decreasing as the point $p$ begins on the $y$ axis and tends to the boundary in either horizontal direction.

But if it is assumed the values of the parameter $a$ stop at, say, $a_{0}<$ 0 , one must work to get a contradiction. The idea is that, although the cap $\Sigma\left(a_{0}\right)$ itself may not have measure smaller than the required value in Proposition 1.3, it is possible by a clever argument to excise a compact subdomain $K$ of $\Sigma\left(a_{0}\right)$ and apply the "thin maximal principle" to the domain $\Sigma\left(a_{0}\right) \backslash K$. This will yield a contradiction to the assumption about $a_{0}$ and so we obtain the result concerning strictly decreasing in the symmetric direction.

We use this method to get the following result.

Proposition 1.4. Assume $\Omega$ is an $S$-C domain in $\mathbb{R}^{2}$ and that it has at least two $S-C$ orientations, say in the $m$ and $M$ directions, with orthogonal convex directions $k$ and $K$ respectively (i.e., the lines of symmetry have directions $m$ and $M$ ). Then the solution of equation (3) has a unique point in the domain where it achieves its maximum.

Proof. Let the notation $D_{V}(u)(P)$ denote the directional derivative of the function $u$ in the $V$ direction and evaluated at a point $P \in \Omega$. The results above indicate that if $P$ is not on the line generated by $m$ then it is strictly decreasing in the convex direction $k$. That is,

$$
D_{k}(u)(P)=\nabla u(P) \cdot k<0,
$$

so the gradient is nonzero. Similarly for $P$ on $M$. Hence only points on $m$ and $M$ can be maximums, and their intersection $P$ satisfies $\nabla u(P)=0$. Note that the maximum point $P$ is not changed if we change the function $f$; it is a property of the domain and not of the forcing function.

\section{Domain With one S-C orientation}

One can easily construct domains in $\mathbb{R}^{2}$ which have only one S-C orientation. Our example is an isosceles triangle $\Lambda$ (which is not equilateral - in this case one has three $\mathrm{S}-\mathrm{C}$ orientations) with base on the $y$ axis and points $A=(0, a), B=(0,-a)$ and $C=(c, 0)$, $c>0$. This is S-C with the $x$ axis as the line of symmetry and the $y$ axis as its associated convex direction. From the above theory we 
know that the solution to (3) on $\Lambda$ must have any maximum points on the real axis segment $0<x<c$. We also know, by symmetry and the moving plane method that

$$
\begin{array}{lll}
u_{y}>0 & \text { for } & y<0 \\
u_{y}<0 & \text { for } & y>0
\end{array}
$$

and that $u_{x}>0$ near the $y$ axis and $u_{x}<0$ near c.

Now writing the angle at the vertex $A$ as $2 \alpha$ (and also at $B$ ), we construct a four-sided figure (the sides are all line segments) as follows. Draw the line from $A$ by bisecting the angle at $A$. The line meets the side $B C$ at a point, say $Q$. The segment $A Q$ together with the segments $B Q$ and $A B$ form a triangle $\Gamma$. Reflect $\Gamma$ along the line $A Q$, so that the line $Q B$ reflects along $Q T$ The four-sided figure has vertices at $A, B, Q, T$, and lies in $\Lambda$. We replace the original domain $\Lambda$ by this domain $\Omega=[A, B, Q, T]$, a subdomain.

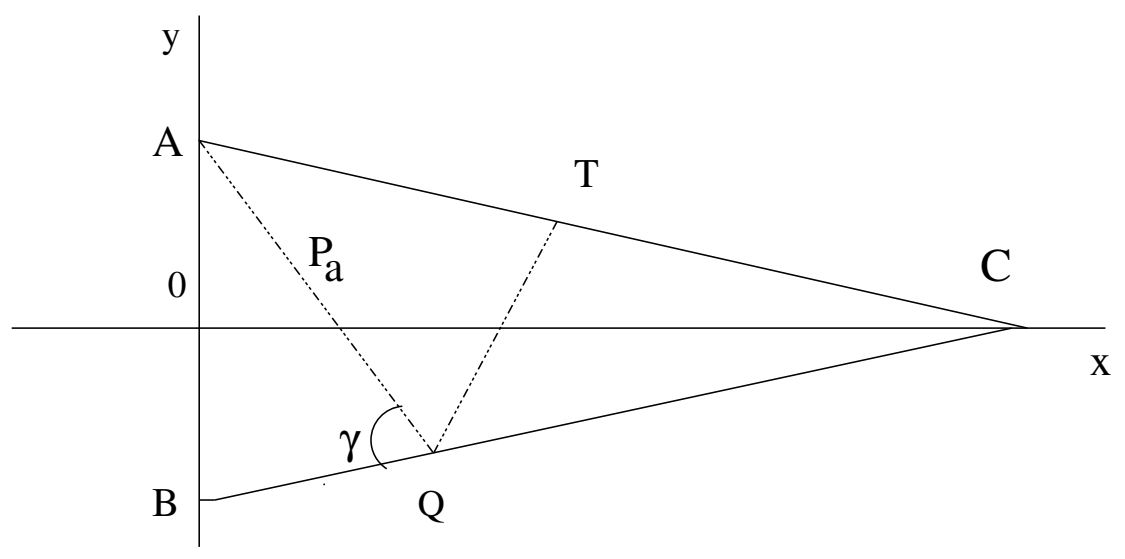

In our case we are considering the domain $\Omega$ and lines orthogonal to the segment $A Q$ which is the line of symmetry. The convex direction is the direction orthogonal to the segment $A Q$. Using the technique above we have the $L_{0}$ line given by $A Q$ and beginning with the point $B$ we select lines $L_{a}$ parallel to $L_{0}$. For each admissible choice of the parameter $a$, we construct a cap $\Sigma(a)$ and define for each $P$ in the cap, with one vertex at $B$, determined by the line $L_{a}$, the function

$$
w((x, y): a)=u(P)-u\left(P^{\perp}\right),
$$

where $P^{\perp}$ is the reflection of $P$ in $L_{a}$. Of course, the triangle determined by this value of $a$ has its reflection in the line $L_{a}$ inside $\Omega \subseteq \Lambda$. Thus two of the sides of $\Sigma(a)$ are parts of the boundary of 
$\Lambda$ and the remaining two sides lie in $\Omega$. Thus $w(\cdot: a)$ is well defined and satisfies $\Delta w+\gamma w=0$ with $\gamma$ defined by equation (5). In checking the required inequality on the boundary of $\overline{\Sigma(a)}$, we have $w(\cdot: a)=0$ on the part of the line $L_{a}$ that lies within $\overline{\Sigma(a)}$, and $w(\cdot: a)<0$ on the other two sides. Hence, by the process outlined above, we see that for each admissible $a$ up to and including $a=0$ we have $w(P: a)<0$ in each such domain. Hence, $u(P)<u\left(P^{\perp}\right)$ for all $P$ in $\Sigma(a)$.

We recall that the point where the line segment $A Q$ crosses the $x$ axis is the center of the largest inscribed circle that can be placed inside of the triangle $A B C$. Label this point $P_{0}=\left(x_{0}, 0\right)$. We also have the following.

Proposition 2.1. The function $u$ is strictly increasing on the segment $\left[(x, 0): 0<x<x_{0}\right]$.

Proof. Let points $P_{1}=\left(x_{1}, 0\right)$ and $P_{2}=\left(x_{2}, 0\right)$ be given with $0<$ $x_{1}<x_{2}<x_{0}$. Starting with the line $L_{a}$ through $P_{1}$ and moving the line continuously toward the parallel segment $A Q$, we note the following. The reflection is a continuous process in the parameter $a$. Also, if we write $P_{j}(P)$ as the projection of the point $P$ onto the real axis, it is also a continuous process. Let $P_{2}=P_{j}\left(P_{1}^{\perp}\right)$ where $P_{1}^{\perp}$ is the unique point which is the reflection of the given point $P_{1}$ and a new line $L_{a^{\prime}}$. There is a unique choice of $a^{\prime}$ that will do this. We know from the information about $u$ in the original triangle $\Lambda$ that

$$
u\left(P_{1}^{\perp}\right)<u\left(P_{j}\left(P_{1}^{\perp}\right)\right)=u\left(P_{2}\right) .
$$

Thus

$$
u\left(P_{1}\right)<u\left(P_{1}^{\perp}\right)<u\left(P_{j}\left(P_{1}^{\perp}\right)\right)=u\left(P_{2}\right) .
$$

This completes the proof.

Remark. If we examine the $C^{2}$ expansion of $u$ at $P_{0}$, we find, for $x^{\prime}=x_{0}+\varepsilon$ and $x^{\prime \prime}=x_{0}-\varepsilon$,

$$
u\left(x^{\prime}, 0\right)=u\left(x_{0}, 0\right)+u_{x}\left(x_{0}, 0\right)(\varepsilon)+\mathcal{O}\left(\varepsilon^{2}\right) .
$$

Similarly,

$$
u\left(x^{\prime \prime}, 0\right)=u\left(x_{0}, 0\right)+u_{x}\left(x_{0}, 0\right)(-\varepsilon)+\mathcal{O}\left(\varepsilon^{2}\right) .
$$

Subtracting these two equations and using the ideas of reflections above, we have

$$
0<u\left(x^{\prime}, 0\right)-u\left(x^{\prime \prime}, 0\right)=u_{x}\left(x_{0}, 0\right)(2 \varepsilon)+\mathcal{O}\left(\varepsilon^{2}\right) .
$$


Thus the "sign" of the right side of this equation is determined by the first term. Since $\varepsilon>0$ we have that $u_{x}\left(P_{0}\right)>0$.

Note that we could have begun this process from the vertex $B$ with appropriate similar conclusion in a "different part" of triangle $A B C$.

We note at this time that, in addition to the above two constructions, there is also another simple subdomain of triangle $A B C$ to which we can apply this process. To wit, let the point $P_{m}=\left(\frac{c}{2}, 0\right)$ be the mid point of the line joining the origin to the point $(c, 0)$. Now we can consider the subdomain of the original triangle determined by the part of the sides $A C$ and $B C$ from the points $\left(\frac{c}{2}, \pm y_{m}\right)$ where the line $x=\frac{c}{2}$ meets these sides. The symmetry line is the line through $\frac{c}{2}$ joining the points determined by $\left(\frac{c}{2},+y_{m}\right)$ and $\left(\frac{c}{2},-y_{m}\right)$. Let the triangle determined by these points and the vertex $(c, 0)$ be labeled $\Delta$. Clearly, reflection of $\Delta$ in the line $L_{\frac{c}{2}}$ remains inside $A B C$ and, applying the technique above, we have for $P^{\perp}$ the reflection of $P$ in the line $x=\frac{c}{2}$, for all $P \in \Delta$,

$$
u(P)<u\left(P^{\perp}\right) .
$$

The following is true.

Proposition 2.2. The function $u(x, 0)$ is strictly decreasing as $x$ increases from $\frac{c}{2}$ to $c$.

Proof. Let $\left(x^{\prime}, 0\right)$ and $\left(x^{\prime \prime}, 0\right)$ be given with $\frac{c}{2}<x^{\prime}<x^{\prime \prime}<c$. The proof is similar to that given in Proposition 2.1 and we omit the details.

It is also true (by a discussion similar to that for the expansion of $u$ near $\left.P_{0}\right)$ that $u_{x}\left(\frac{c}{2}\right)<0$.

At this stage we have shown that any maximum for $u$ can only occur between the points $P_{0}$ and $P_{m}$ on the real axis.

\section{Numerical information on the LOCATION OF THE MAXIMA}

Although we have given some estimates, based on the geometry of our given triangle $A B C$, as to where a maximum may occur, we have not been successful in proving that such an (absolute) maximum is unique or its exact location. The numerical data following implies that indeed the maximum point is unique and, moreover, its position 
depends only on the geometry of the triangle and not on the function $f(z)$. From our point of view this is surprising.

We constructed a pseudo-triangle $A B C$ consisting of a lattice of square cells from -15 to 15 on the $y$-axis, with variable geometry allowing up to 90 cells on the $x$-axis. The location of vertex $C$ is given by $(c, 0)$. When that information is given, the program initiates the computation by giving an initial value of $u=0.1$ to all points $(i, j)$ such that $|j| \leq \operatorname{int}\left[15\left(1-\left(\frac{i}{c}\right)\right)\right], 0 \leq i \leq c$ and giving a value $u=0$ to all other points in the grid, so that the boundary grid points are zero. Observe that this pseudo-triangle is an $\mathrm{S}-\mathrm{C}$ domain.

The Laplacian of $u$ is approximated by the average of the four adjacent grid points and added to $f(u)$, giving

$u_{\text {new }}(i, j)=\frac{1}{4}\{u(i+1, j)+u(i-1, j)+u(i, j+1)+u(i, j-1)\}+f(u(i, j))$,

where $(i, j)$ is the grid point where $u$ is evaluated. The results are stored in a new matrix of grid points $u_{\text {new }}$ for the next iteration. All boundary grid points are kept at zero. Finally, the sum of the squares of the differences between the new and old values is obtained, and when its square root is less than a given tolerance, the iterations are stopped and the maximum grid point values are located.

We used three different $f(u): f_{1}(u)=.03 u^{2}, f_{2}(u)=.01\left(1+u^{2}\right) / 4$, and $f_{3}(u)=.005 u(1+u)$. We ran the program for different values for $c$ between $20 \leq c \leq 90$. In all cases examined, the maximum values (though different) occurred in the same place, $u(x, 0)$, where the $x$-value is shown in the table:

\begin{tabular}{|l|r|r|r|r|r|r|r|r|r|}
\hline$c$ & 20 & 26 & 30 & 40 & 50 & 60 & 70 & 80 & 90 \\
\hline$x$ & 7 & 8 & 9 & 11 & 12 & 14 & 15 & 16 & 17 \\
\hline
\end{tabular}

Different tolerances were used: $10^{-4}$ for $f_{1}$ and $f_{2}$, and $10^{-9}$ for $f_{3}$, but the location of the maximum did not change for all three functions. The choice of $c=26$ is an attempt to make the pseudotriangle $A B C$ as close as possible to an equilateral triangle, where the center of the inscribed circle is located at $x=5 \sqrt{3} \approx 8.66$, so an $x$ value of 8 in the table is a reasonable approximation to the maximum point's location. 


\section{REFERENCES}

[1] Berestyski, H. and Nirenberg, L., On the method of moving planes and the sliding method, Bal. Soc. Brasil. Mat. (N.S.) 22 (1991) 1-37.

[2] Berestyski, H., Nirenberg, L. and Varadhan, S.R.S., The principal eigenvalue and maximum principle for second-order elliptic operators in general domains, Comm. Pure and Applied Math., 47 (1994) 47-92.

[3] Du, Y., Order structure and topological methods in nonlinear partial differential equations, Vol. 1. World Scientific 2006.

[4] Fraenkel, L.E., Introduction to maximum principles and symmetry in elliptic problems. Cambridge University Press 2000.

[5] Gidas, B., Ni, Wei-Ming and Nirenberg, L., Symmetry and related properties via the maximum principle, Commun. Math. Phys. 66 (1979) 209-243.

Joseph Cima Dr. Cima received his M.A. and Ph.D degrees at Pennsylvania State University. He is a professor of mathematics at the University of North Carolina at Chapel Hill. He has served as Secretary of the American Mathematics Society. His interests are in Several Complex Variable Theory, Operator Theory and Harmonic Analysis.

William Derrick Dr. Derrick received his Ph.D. degree at Indiana University. His research interests are in Complex Analysis, Partial Differential Equations, Applied Mathematics and Modeling. He is currently Professor Emeritus at the University of Montana in Missoula, Montana.

(J. Cima) Dept. of Mathematics, University of North Carolina, Chapel Hill, NC 27599-3250, USA

(W. Derrick) Department of Mathematics, University of Montana, Missoula, Montana 59812, USA

E-mail address, J. Cima: cima@email.unc.edu 